\title{
Pyruvate Carboxylase Supports MCF10A-Ras Cell Survival in Extracellular Matrix Detached Conditions
}

\section{Madeline P Sheeley}

Purdue University https://orcid.org/0000-0002-5102-0533

\section{Violet A Keisel}

Purdue University Center for Cancer Research

Nadia M. Atallah

Purdue University

\section{Shawn S. Donkin}

Purdue University

\section{Stephen D. Hursting}

University of North Carolina at Chapel Hill

\section{Michael K. Wendt}

Purdue University Center for Cancer Research

Dorothy Teegarden ( $\nabla$ teegarden@purdue.edu )

Purdue University

\section{Research Article}

Keywords: breast cancer, pyruvate carboxylase, glutamine metabolism, glucose metabolism, energy metabolism

Posted Date: June 29th, 2021

DOI: https://doi.org/10.21203/rs.3.rs-647480/v1

License: (c) (i) This work is licensed under a Creative Commons Attribution 4.0 International License. Read Full License 


\section{Abstract}

Background: Throughout metastatic progression, cancer cells acquire anchorage independence, or the ability to survive detached from the extracellular matrix (ECM). While untransformed epithelial cells reduce energy metabolism when detached, cancer cells display metabolic flexibility to continue important metabolic processes. Glucose and glutamine are predominant nutrients utilized for energy as well as other purposes, and their metabolism is regulated by cancer cells.

Methods: The purpose of the current studies was to determine the effects of detachment on glucose and glutamine metabolism in human breast epithelial MCF10A cells transfected with the Harvey-ras oncogene (MCF10A-ras), a model of early-stage cancer. Detachment was simulated with poly-HEMA coated plates, and intracellular metabolic flux was determined using stably labeled ${ }^{13} \mathrm{C}_{5}$-glutamine and ${ }^{13} \mathrm{C}_{6}$-glucose tracers.

Results: Results show reduced glutamine flux in detached cells as determined by reduced accumulation of label in glutamate (21\%), malate (30\%), and aspartate $(23 \%)$ from ${ }^{13} \mathrm{C}_{5}$-glutamine. Detachment also reduced flux of ${ }^{13} \mathrm{C}_{6}$-glucose to pyruvate and lactate pools by $51 \%$ and $29 \%$, respectively. Similarly, detachment reduced total intracellular pool sizes of pyruvate (51\%), lactate (49\%), a-ketoglutarate (43\%), fumarate $(32 \%)$, malate $(19 \%)$, alanine (35\%), serine $(35 \%)$, and glutamate $(28 \%)$ compared to attached cells, but citrate and aspartate pool sizes were unchanged. Compared to attached cells, detachment increased pyruvate carboxylase (PC) mRNA abundance and protein expression by $131 \%$ and $190 \%$, respectively. In detachment, PC activity, determined by ${ }^{13} \mathrm{C}_{6}$-glucose derived $\mathrm{M}+3$ isotopomers, was shown to preferentially replenish malate and aspartate, but not citrate pools. In addition, doxycyclineinducible shRNA depletion of PC significantly decreased, while doxycycline-inducible PC overexpression significantly increased, detached cell viability. Further, a switch from glutamine to PC activity for anaplerosis was demonstrated, as supplementation with the cell permeable analog of the tricarboxylic acid cycle intermediate, a-ketoglutarate, a downstream metabolite of glutamine, decreased PC mRNA abundance in detached cells.

Conclusion: Collectively, these results suggest that detached breast cancer cells increase PC activity in response to decreased glutamine-derived anaplerosis to promote cell survival.

\section{Background}

Metastasis accounts for the majority of cancer-related deaths [1]. The five-year survival rate of breast cancer patients is $28 \%$ when diagnosed with metastatic disease compared to a $99 \%$ five-year survival rate in patients with a localized diagnosis [2]. Metastasis is a multistep process that includes cellular modifications in the primary tumor, invasion into surrounding tissue, systemic dissemination, and colonization of a secondary tumor at a distal site [3]. Thus, research that identifies regulators of progression through the metastatic cascade may provide targets to improve the survival of breast cancer patients. 
Survival and growth of normal epithelial cells are dependent on attachment to the extracellular matrix (ECM). In a primary tumor, cellular changes permitting "anchorage independence," which enables cancer cell survival when detached from the ECM, promotes tumor growth and metastasis [4]. In nontransformed cells, integrins mediate attachment to the matrix and, upon detachment, initiate signaling that stimulates anoikis, a form of programmed cell death [5]. For example, integrins reduce activation of focal adhesion kinase (FAK), leading to reduced downstream signaling through pro-survival signaling pathways such as mitogen activating protein kinase (MAPK) and phosphoinositide-3 kinase (PI3K) [6]. Oncogenic transformation, such as those mediated by mutation to RASor amplification of ERBB2, assists cancer cells in gaining anchorage independence which enables their survival when detached from the ECM by maintaining activation of survival signaling pathways such as PI3K $[7,8]$. Thus, oncogeneic transformation sustains detached cell viability through stimulation of signaling pathways, which confer inhibition of anoikis and alterations in cellular metabolism.

Reprogrammed energy metabolism is an emerging hallmark of cancer [9], and evidence supports that metabolic reprogramming plays an important role in maintaining survival of ECM-detached cancer cells. For example, nonmalignant epithelial cells decrease energy metabolism pathways upon detachment [4]. However, detached MCF10A human breast epithelial cells with overexpression of the ERBB2 oncogene increased glucose and glutamine flux into the tricarboxylic acid (TCA) cycle compared to detached untransformed MCF10A cells [10]. Additionally, overexpression of FAK increased glycolysis in detached pancreatic ductal adenocarcinoma cells [11]. Further, detached MDA-MB-231 breast cancer cells increased the expression of antioxidant enzymes, which was required for fatty acid oxidation and the subsequent production of adenosine triphosphate (ATP) [12]. These results suggest that oncogenic transformation enables adaptations in cellular energy metabolism in ECM detached conditions.

The anaplerotic enzyme, pyruvate carboxylase (PC), is responsible for the conversion of pyruvate to oxaloacetate to replenish the TCA cycle. Previous results demonstrate that PC is required for breast to lung metastasis in vivo [13]. In addition, Phannasil et al. demonstrated that PC activity contributes to metastatic steps such as migration and invasion [14]. Interestingly, a preference for PC over glutamine anaplerosis was previously identified in breast to lung metastatic tumors, suggesting a "PC switch" in metastatic progression to adjust to nutrient availability [15]. PC activity replenishes the TCA cycle and is linked to increased fatty acid synthesis and oxidative stress protection in breast cancer models $[16,17]$. While PC activity is utilized by cancer cells to supply substrate for various metabolic processes, its role in detached conditions has not been established. In the current work it was hypothesized that PC is required to maintain cell viability of transformed breast epithelial cells by replenishing the oxaloacetate pool to rescue cells from decreased glutamine availability in ECM detached conditions.

\section{Methods}

\section{Cell Culture}


MCF10A cells transfected with the Harvey-ras oncogene (MCF10A-ras), a of model early-stage breast cancer, were a gift from Michael Kinch, Purdue University. Cells were grown at $37^{\circ} \mathrm{C}$ with $5 \% \mathrm{CO}_{2}$ in Dulbecco's Modified Eagle Medium (DMEM, Sigma, St. Louis, MO) containing $5 \mathrm{mM}$ glucose, $2.5 \mathrm{mM}$ glutamine, and no sodium pyruvate. Media also contained $5 \%$ horse serum (Gibco, Waltham MA), $1 \%$ penicillin/streptomycin (Gibco), $10 \mathrm{mg} / \mathrm{L}$ insulin (Sigma), $50 \mu / \mathrm{L}$ cholera toxin (Sigma), $20 \mu \mathrm{g} / \mathrm{L}$ epidermal growth factor (Sigma, St. Louis, MO), and $50 \mathrm{mg} / \mathrm{L}$ hydrocortisone (Sigma).

\section{Poly-HEMA-coated plates}

Extracellular matrix (ECM) detachment was simulated using Poly(2-hydroxyethyl methacrylate) (PolyHEMA, Sigma) coated plates. Plates were coated with $70 \mu \mathrm{L} / \mathrm{cm}^{2}$ of $20 \mathrm{mg} / \mathrm{mL}$ Poly-HEMA in $95 \%$ ethanol. The plates were dried overnight under ultraviolet light after the addition of Poly-HEMA. The PolyHEMA coated plates and dishes were rinsed twice with sterile $1 \mathrm{x}$ calcium/magnesium-free phosphatebuffered saline (PBS) before use.

\section{MTT assay}

Viability assays were performed according to the manufacturer's instructions using 3-(4,5dimethylthiazol-2-yl)-2,5-diphenyltetrazolium bromide (MTT, Sigma). Briefly, cells were plated at 20k cells/well with indicated treatment into Poly-HEMA coated 96-well plates. MTT solution was added to each well to a final concentration of $1 \%$ for two hours, followed by solubilization in dimethylsulfoxide and absorbance measured at $570 \mathrm{~nm}$.

\section{RNA isolation and analysis}

Cells were plated into 100 mm Poly-HEMA coated dishes. After 40 hours, Tri-Reageant (Molecular Research Center, Cincinnati, $\mathrm{OH}$ ) was used to harvest and isolate RNA according to the manufacturer's instructions. RNA was reverse transcribed using the MMLV reverse transcriptase (Promega, Madison, $\mathrm{WI}$ ). Real-time quantitative PCR was performed on the subsequent cDNA using a LightCycler 480 instrument using LightCycler 480 SYBR Green I Master Mix (Roche, Indianapolis, IN). All target gene mRNA abundance was normalized to $18 \mathrm{~S}$ abundance with the comparative $\mathrm{Ct}$ method $\left(2^{-\Delta \mathrm{Ct}}\right)$. 
Table 1

Primers used for qRT-PCR.

\begin{tabular}{|c|c|}
\hline $18 S$ & Forward: 5'- TTAGAGTGTTCAAAGCAGGCCCGA-3' \\
\hline & Reverse: 5'- TCTTGGCAAATGCTTTCGCTC-3' \\
\hline ASCT2 & Forward: 5'- TGAACATCCTGGGCTTGGTAG-3' \\
\hline & Reverse: 5'- AGCAGGCAGCACAGAATGTAC-3' \\
\hline GLUD1 & Forward: 5'- TTAGAGTGTTCAAAGCAGGCCCGA-3' \\
\hline & Reverse: 5'- TCTTGGCAAATGCTTTCGCTC-3' \\
\hline GOT1 & Forward: 5'-CAACTGGGATTGACCCAACT - 3' \\
\hline & Reverse: 5'- GGAACAGAAACCGGTGCTT - 3' \\
\hline GOT2 & Forward: 5'-ACCCATGTGGAAATGGGACC - 3' \\
\hline & Reverse: 5'- GACTTCGCTGTTCTCACCCA-3' \\
\hline GLUT1 & Forward: 5'-TATCGTCAACACGGCCTTCACTGT - 3' \\
\hline & Reverse: 5'- CACAAAGCCAAAGATGGCCACGAT - 3' \\
\hline HK2 & Forward: 5'-CTGCAGCGCATCAAGGAGAACAAA - 3' \\
\hline & Reverse: 5'- ACGGTCTTATGTAGACGCTTGGCA - 3' \\
\hline LDHA & Forward: 5'- TGGTCCAGCGTAACGTGAACATCT - 3' \\
\hline & Reverse: 5'-TTGCAACCGCTTCCAATAACACGG - 3' \\
\hline PC & Forward: 5'- ATGTTGCCCACAACTTCAGCAAGC - 3' \\
\hline & Reverse: 5'- AGTTGAGGGAGTCAAACACACGGA-3' \\
\hline
\end{tabular}

\section{Glutamine and glucose flux analysis}

Following 40 hours in attachment or detachment, media was changed to media containing either $100 \%$ $(2.5 \mathrm{mM}){ }^{13} \mathrm{C}_{5}$-glutamine or $100 \%(5 \mathrm{mM}){ }^{13} \mathrm{C}_{6}$-glucose. Cells were incubated at $37^{\circ} \mathrm{C}$ for two hours, followed by harvest into $70 \%$ ethanol at $70^{\circ} \mathrm{C}$ and the addition of $1 \mu \mathrm{g}$ of the internal standard norvaline to each sample. Intracellular metabolites were extracted from the cells into ethanol by a 5-minute incubation at $95^{\circ} \mathrm{C}$. A bicinchoninic acid (BCA, ThermoFisher, Waltham, MA) assay was used to measure protein content in the cell pellet, and the supernatant dried. Methoxylamine hydrochloride in pyridine was used to derivatize metabolites as previously described [18]. Following derivatization, metabolites were analyzed with gas chromatography-mass spectrometry (GC-MS) using a TG-5MS gas chromatography column and Thermo TSQ 8000 triple quadrupole mass spectrometer. Mass spectra data were acquired using Chromeleon 7 software (ThermoFisher). Intracellular pool sizes were calculated by dividing the area under the curve for the total metabolite by norvaline and protein in the cell pellet to control for recovery 
and cell quantity, respectively. Isotopomer percent enrichment of the metabolite pool sizes was used to calculate ${ }^{13} \mathrm{C}$ flux, and results are calculated relative to levels determined for attached cells.

\section{Western blot analysis}

Following two washes with calcium/magnesium-free PBS, cells were harvested on ice into $1 \mathrm{x}$ radioimmunoprecipitation assay (RIPA) lysis buffer (Cell Signaling, Danvers MA) supplemented with 1\% phosphatase (Sigma) and 1\% phenylmethylsulfonyl fluoride protease inhibitors (Cell Signaling). To harvest detached cells, cells were pelleted by 5-minute centrifugation at 1000 RPM and rinsed twice with calcium/magnesium-free PBS. Pelleted detached cells were resuspended in $25 \mu \mathrm{L}$ of RIPA lysis buffer. Attached and detached cells were sonicated for 15 minutes and centrifuged for 15 minutes at 12,000 revolutions per minute. The supernatant was collected and protein quantified using the BCA assay. A 4$15 \%$ gradient polyacrylamide gel (Bio-Rad Laboratories, Hercules, CA) was used to resolve $20 \mu \mathrm{g}$ of protein by electrophoresis. Primary antibodies for PC and actin were purchased from Sigma. Proteins were transferred to a nitrocellulose membrane (Bio-Rad Laboratories) and antigen-antibody complexes were detected by the Li-Cor Odyssey imaging system.

\section{PC knockdown and overexpression}

Doxycycline (Dox) inducible TRIPZ lentiviral human shRNA which targeted PC and was purchased from GE Dharmacon (Lafayette, CO) and Dox inducible lentiviral plasmid containing the human PC gene (NM_000920.2) was purchased from VectorBuilder (Chicago, IL). Polyethylamine was used to transduce HEK293T cells with lentiviral plasmids and the lentiviral particles harvested. MCF10A-ras cells were transfected for 48 hours with $10 \mu \mathrm{g} / \mathrm{mL}$ polybrene, and stably transfected cells were selected using puromycin $(5 \mathrm{mg} / \mathrm{mL})$. For PC overexpression, hygromycin $(450 \mathrm{ug} / \mathrm{mL})$ was used in addition to puromycin to select for stable transfection. PC knockdown or overexpression was verified by PCR analysis of PC mRNA abundance following treatment with Dox $(0.5 \mathrm{ug} / \mathrm{mL})$ for three days.

\section{Statistical analysis}

Results are expressed as mean \pm S.E.M. Comparisons between two groups were determined using an independent samples $t$-test, or across groups by analysis of variance (ANOVA). Statistical significance of $P<0.05$.

\section{Results}

\section{Detached MCF10A-ras Cell Survival}

To evaluate the response of the MCF10A-ras cells to ECM detachment, cell viability was measured over a time course of $0,24,40$, and 48 hours (Fig. 1A). Cell viability decreased with time in detached conditions up to 40 hours, with an increase at 48 hours, suggesting growth of the surviving detached cells after 40 hours. Given that 40 hours was the time point with the lowest level of viability, this time point was selected for further study. 
Because previous studies demonstrate the importance of energy metabolism for cell survival in ECM detached conditions $[10,19]$, the dependence of detached MCF10A-ras cells on glutamine and glucose was determined. Depriving cells of either glutamine or glucose decreased detached cell viability by $57 \%$ or $69 \%$, respectively, and deprivation of both glucose and glutamine decreased cell viability by $82 \%$ (Fig. $1 \mathrm{~B}$ ). These results demonstrate a dependence on glutamine or glucose for the survival of MCF10A-ras cells in detachment.

\section{Effects of Detachment on Glutamine Metabolism}

Due to the dependence on glutamine for survival in detached conditions, the metabolism of glutamine in detached cells was determined. Compared to attached cells, detached cells had decreased mRNA levels of the glutamine transporter, Alanine/Serine/Cysteine-preferring Transporter 2 (ASCT2) (Fig. 2C). Flux

analysis of ${ }^{13} \mathrm{C}_{5}$-glutamine showed that detachment decreased glutamine flux into glutamate, malate, and aspartate, demonstrating reduced TCA cycle anaplerosis from glutamine (Fig. 2A and 2B). In addition, In detached cells, lower mRNA abundance relative to attached cells of enzymes involved in the metabolism of glutamate, which is directly metabolized from glutamine, were also shown, including glutamate dehydrogenase (GLUD1) and the cytosolic glutamate oxaloacetate transaminase (GOT) 1 (Fig. 2C). In contrast, mRNA abundance of the mitochondrial isoform GOT2 was unchanged in detached cells (Fig. 2C). These results support that there is a decrease in glutamine uptake and catabolism in detached MCF10A-ras cells compared to attached cells.

\section{Effects of Detachment on Glucose Metabolism}

To explore glucose metabolism in ECM detached conditions, the effects of detachment on glycolysis were determined. Intracellular pool sizes of the end products of glycolysis, pyruvate, and lactate, were both decreased in detachment (Fig. 3A). Universally labeled ${ }^{13} \mathrm{C}_{6}$-glucose flux, which results in pyruvate and lactate labeled with three ${ }^{13} \mathrm{C}$ 's $(M+3)$, were both decreased following detachment (Fig. 3B). Decreased mRNA abundance of glycolytic enzymes hexokinase 2 (HK2) and lactate dehydrogenase A (LDHA) was observed in detached cells relative to attached cells (Fig. 3C). However, compared to attached cells, mRNA abundance of glucose transporter 1 (GLUT1) was unchanged following detachment (Fig. $3 \mathrm{C}$ ). Similar to glutamine metabolism, these results demonstrate that while glucose is required for detached cell survival, detached cells decrease glucose metabolism through glycolysis compared to their attached counterparts.

Given the decrease in glycolysis observed in detached cells, it was hypothesized that glucose flux into the TCA cycle was also decreased. Compared to attached cells, detachment decreased pool sizes for all intermediates measured, except citrate which was unchanged (Fig. 3D). Additionally, free amino acid pool sizes which are metabolically connected to glutamine, glucose, or TCA cycle metabolism were measured. Detachment decreased total pool sizes for alanine, serine, and glutamate, but no change was observed for aspartate (Fig. 3E). Furthermore, glucose-derived anaplerosis through pyruvate dehydrogenase was measured, using ${ }^{13} \mathrm{C}_{6}$-glucose flux into citrate which results in $\mathrm{M}+2$ labeling, was decreased in detached cells relative to attached (Fig. 3F). Collectively these results suggest that energy metabolism through 
glycolysis and the TCA cycle is reduced following detachment. Overall, these results suggest that detachment suppresses TCA cycle anaplerosis from both glutamine and glucose. However, these results also suggest that detached MCF10A-ras cells maintain the production of citrate and aspartate in detachment.

\section{Detachment Increases Pyruvate Carboxylase}

Replenishment of the oxaloacetate pool to support the TCA cycle can be mediated by PC activity, which synthesizes oxaloacetate from pyruvate and bicarbonate. Because pool sizes of the downstream metabolites of oxaloacetate, citrate and aspartate, were unchanged following detachment, PC activity was assessed. Compared to attached cells, detached cells significantly increased PC mRNA abundance (Fig. 4A) and protein expression (Fig. 4B). Further, the flux of ${ }^{13} \mathrm{C}_{6}$-glucose to $\mathrm{M}+3$ labeled malate and aspartate was significantly higher in detached cells, demonstrating an increase in PC activity. However, there was significantly decreased flux to $M+3$ citrate in detached cells (Fig. 4C). These data demonstrate a preferential utilization of PC-derived oxaloacetate into malate and aspartate, rather than citrate, in detached cells. These results suggest that in detached cells there is reduced forward TCA cycle flux that would replenish oxaloacetate from non-PC sources, and are consistent with the decreased glutamine flux into the TCA cycle.

\section{PC is Required for Detached Cell Survival}

Due to the increase in PC activity and shift in glucose utilization, the requirement of PC activity for detached cell survival was assessed. Dox treatment of attached dox-inducible shPC MCF10A-ras cells reduced PC mRNA abundance by $73 \%$ (Fig. 5A). PC depletion significantly decreased detached cell viability by $13 \%$ (Fig. $5 B$ ). Treatment of PC-depleted cells with exogenous oxaloacetate ( $2 \mathrm{mM})$, the product of the PC reaction, rescued cell viability to a level similar to that of PC-expressing cells (Fig. 5B). In addition, treating PC-depleted cells with $2.5 \mathrm{mM}$ aspartate, a downstream metabolite of oxaloacetate produced via mitochondrial GOT2 activity, also rescued viability to that of PC-expressing cells (Fig. 5C).

Previous work demonstrated that metastatic MCF10CA1 a cells have five-fold higher PC expression than MCF10A-ras cells [16]. Given the increased PC expression observed in cells with higher metastatic capability than the MCF10A-ras cells and the requirement for anchorage independence in metastatic cells, the effect of PC overexpression in MCF10A-ras cells on cell viability in detached conditions was assessed. Results demonstrate that overexpressing PC in MCF10A-ras cells significantly increased their viability in detached conditions compared to detached control cells (Fig. 6A and 6B).

A recent study identified that glutamine metabolism is required for the survival of detached liver cancer cells [20], and a PC "switch" has been proposed to describe the metabolic flexibility of cancer cells where depletion of glutamine from the microenvironment increases PC activity [21]. To test whether a switch to PC-mediated anaplerosis due to reduced glutamine availability occurs in detachment, detached cells were treated with a membrane-permeable analog of a-ketoglutarate, dimethyl-a-ketoglutarate (DM-aKG), and PC mRNA levels measured. Treatment with DM-aKG, which is the downstream metabolite of glutamine 
that enters the TCA cycle, decreased PC mRNA abundance in detached cells (Fig. 7A). These data support that detachment in MCF10A-ras cells reprograms cell metabolism to suppress glutamine flux into the TCA cycle and "switch" to preferential utilization of PC for TCA cycle anaplerosis.

\section{Discussion}

Metastasis of cancer cells is a multistep process, which includes survival in ECM detachment [6]. Thus, tumor cells that demonstrate anchorage-independent growth possess a survival advantage which may promote their spread to distant sites. Detachment from ECM in non-transformed epithelial cells induces cellular changes that suppress flux of metabolites, including glucose and glutamine, into energyproducing pathways such as glycolysis and the TCA cycle which contributes to reduced viability in ECM detached conditions [4]. Compared to non-transformed cells, oncogenic transformation rescues glucose and glutamine flux into the TCA cycle in detached conditions [10]. The flux of metabolites through the TCA cycle generates the coenzymes nicotinamide adenine dinucleotide or flavin adenine dinucleotide, which are then oxidized at the electron transport chain for ATP production, collectively suggesting that enhanced flux of nutrients through the TCA cycle contributes to anchorage independence in transformed cells. Additionally, intermediates of the TCA cycle are utilized by cells to mediate biosynthetic reactions required for cell proliferation, such as amino acids and lipids, suggesting that nutrient metabolism serves additional functions beyond ATP production. The present study demonstrates that detached MCF10A-ras cells display decreased glucose metabolism through glycolysis and decreased anaplerosis from glutamine compared to attached cells. However, detached cells specifically display increased flux of pyruvate, the end product of glycolysis, to oxaloacetate through upregulated expression and activity of PC.

Previous studies identified that PC is required for breast cancer metastasis to the lung. For example, in an in vivo model of breast cancer, metastatic lesions at the lung contained high levels of PC expression and activity [15]. Additionally PC was required for breast to lung metastasis in an animal model [13]. The organotropic role of PC in breast to lung metastasis is consistent with previous literature showing a cellular requirement for PC activity in primary lung tumors [22]. Given the requirement for PC in both primary and metastatic cancer cell growth in the lung, these data may highlight PC as an attractive therapeutic target. The present work identified a novel mechanism by which detached breast cancer cells reprogram energy metabolism to increase the expression and activity of PC. Further, depletion of PC in detachment impairs cell survival, suggesting a specific molecular mechanism by which PC mediates breast to lung metastasis. Additionally, overexpression of PC, a characteristic of metastatic breast cancer $[14,16]$, improves detached viability of MCF10A-ras breast epithelial cells. Collectively, these results suggest that increased PC expression may promote metastasis by supporting survival in ECM detached conditions throughout the metastatic cascade.

Metabolic flexibility has recently been identified as a contributor to the successful metastasis of cancer cells [23]. As cancer cells progress through metastasis, they require adaption to changing nutrient conditions in variable microenvironmental conditions. As a compensatory mechanism, PC may play a 
crucial role in conferring metabolic flexibility to cancer cells. For example, in healthy BALB/c mice, the ratio of pyruvate/glutamine in the lung interstitial fluid is three times higher than in serum [15]. This varying ratio of pyruvate/glutamine may require cancer cells to switch from utilizing glutamine as a means of replenishing the TCA cycle to increasing anaplerosis through PC activity for successful colonization. Indeed, PC activity was increased in lung metastases compared to primary breast tumors [15]. In the current study, a PC switch was demonstrated in detached cells as detachment resulted in decreased glutamine incorporation into the TCA cycle and increased flux of glucose into aspartate and malate pools through PC activity. Further support for metabolic reprogramming in favor of PC over glutamine was demonstrated by the decreased mRNA abundance of PC following the addition of DMaKG to detached cells. These results demonstrate that replenishing the TCA cycle with a metabolite of glutamine reverses the increase in PC abundance. Therefore, detached cancer cells display metabolic flexibility by increasing PC expression and activity in response to reduced glutamine metabolism.

PC's role in aspartate biosynthesis has been well documented in cancer models. In renal cell carcinoma with succinate dehydrogenase (SDH) ablation, the addition of aspartate rescues cells from PC depletion [24]. Consistent with these results, SDHA or SDHB inhibition resulted in an increase in PC activity in both neuroendocrine and prostate cancer cells $[25,26]$. In both of these models, increased PC activity following SDH inhibition resulted in the replenishment of aspartate. Thus, inhibition of the forward TCA cycle by inhibition of SDH activity increases PC-mediated TCA cycle anaplerosis. Consistent with this, the current study identified increased PC activity in detached cells indicated by enrichment of ${ }^{13} \mathrm{C}_{6}$-glucose derived $M+3$ labeling patterns in the intracellular pools of aspartate and malate. These results suggest that detached cells use PC activity to compensate for an inability to synthesize oxaloacetate from glutamine through the forward TCA cycle.

In summary, these findings demonstrate that detachment of MCF10A-ras cells leads to a decreased flux of glucose and glutamine into the TCA cycle compared to their attached counterparts. However, PC expression and activity is increased in detached cells, and this effect is reversed with exogenous addition of the TCA cycle intermediate DM-aKG. These results indicate that PC plays a critical role in promoting the survival of cells in matrix detached conditions and highlights a potential therapeutic target for the prevention of cancer metastasis.

\section{Abbreviations}

aKG a-ketoglutarate

ANOVA Analysis of variance

ASCT2 Alanine/Serine/Cysteine-preferring Transporter 2

Asp Aspartate

ATP Adenosine triphosphate 


\begin{tabular}{|c|c|}
\hline BCA & Bichinchoninic acid \\
\hline DM-aKG & Dimethyl-a-ketoglutarate \\
\hline DMEM & Dulbecco's modified eagle medium \\
\hline Dox & Doxycycline \\
\hline ECM & Extracellular matrix \\
\hline FAK & Focal adhesion kinase \\
\hline GC-MS & Gas chromatography-mass spectrometry \\
\hline GLUD1 & Glutamate dehydrogenase \\
\hline GLUT1 & Glucose transporter 1 \\
\hline GOT & Glutamate oxaloacetate transaminase \\
\hline HK2 & Hexokinase 2 \\
\hline ILK & Integrin linked kinase \\
\hline LDHA & Lactate dehydrogenase $\mathrm{A}$ \\
\hline MAPK & Mitogen activating protein kinase \\
\hline MTT & 3-(4,5-dimethylthiazol-2-yl)-2,5-diphenyltetrazolium bromide \\
\hline OAA & Oxaloacetate \\
\hline PBS & Phosphate-buffered saline \\
\hline PC & Pyruvate carboxylase \\
\hline $\mathrm{PI} 3 \mathrm{~K}$ & Phosphoinositide-3 kinase \\
\hline Poly-HEMA & Poly(2-hydroxyethyl methacrylate) \\
\hline RIPA & Radioimmunoprecipitation assay \\
\hline SDH & Succinate Dehydrogenase \\
\hline TCA & Tricarboxylic acid \\
\hline
\end{tabular}


Ethics approval and consent to participate: Not applicable.

Consent for publication: Not applicable.

Availability of data and materials: Data sharing is not applicable to this article as no datasets were generated or analyzed during the current study.

Competing interests: The authors declare that they have no competing interests.

Funding: This work was supported by the Purdue University Center for Cancer Research; Indiana Clinical Translational Science Institute NIH/NCRR [\#TR000006] and the National Institute of Health [R01CA232589 and R35CA197627].

Authors' contributions: MPS and DT designed experiments and wrote the manuscript. MPS conducted experiments and analyzed the data. NMA and SSD contributed to flux experiments. All authors contributed to the design of experiments as well as editing and reviewing content of the manuscript, and approved the final manuscript.

\section{Acknowledgements}

The authors acknowledge the use of the facilities of the Bindley Bioscience Center, a core facility of the $\mathrm{NIH}-$ funded Indiana Clinical and Translational Sciences Institute

\section{References}

1. Dillekås H, Rogers MS, Straume O. Are $90 \%$ of deaths from cancer caused by metastases? Cancer Med. 2019;8(12):5574-6.

2. Siegel RL, Miller KD, Fuchs HE, Jemal A. Cancer Statistics, 2021. CA Cancer J Clin. 2021;71(1):7-33.

3. Melzer C, von der Ohe J, Hass R. Breast Carcinoma: From Initial Tumor Cell Detachment to Settlement at Secondary Sites. Biomed Res Int. 2017;2017:8534371.

4. Grassian AR, Coloff JL, Brugge JS. Extracellular matrix regulation of metabolism and implications for tumorigenesis. Cold Spring Harb Symp Quant Biol. 2011;76:313-24.

5. Schwartz MA. Integrins, oncogenes, and anchorage independence. J Cell Biol. 1997;139(3):575-8.

6. Paoli P, Giannoni E, Chiarugi P. Anoikis molecular pathways and its role in cancer progression. Biochim Biophys Acta. 2013;1833(12):3481-98.

7. Kang JS, Krauss RS. Ras induces anchorage-independent growth by subverting multiple adhesionregulated cell cycle events. Mol Cell Biol. 1996;16(7):3370-80.

8. Grassian AR, Schafer ZT, Brugge JS. ErbB2 stabilizes epidermal growth factor receptor (EGFR) expression via Erk and Sprouty2 in extracellular matrix-detached cells. J Biol Chem. 2011;286(1):7990.

9. Hanahan D, Weinberg RA. Hallmarks of cancer: the next generation. Cell. 2011;144(5):646-74. 
10. Grassian AR, Metallo CM, Coloff JL, Stephanopoulos G, Brugge JS. Erk regulation of pyruvate dehydrogenase flux through PDK4 modulates cell proliferation. Genes Dev. 2011;25(16):1716-33.

11. Zhang J, Gao Q, Zhou Y, Dier U, Hempel N, Hochwald SN. Focal adhesion kinase-promoted tumor glucose metabolism is associated with a shift of mitochondrial respiration to glycolysis. Oncogene. 2016;35(15):1926-42.

12. Davison CA, Durbin SM, Thau MR, Zellmer VR, Chapman SE, Diener J, Wathen C, Leevy WM, Schafer ZT. Antioxidant enzymes mediate survival of breast cancer cells deprived of extracellular matrix. Cancer Res. 2013;73(12):3704-15.

13. Shinde A, Wilmanski T, Chen H, Teegarden D, Wendt MK. Pyruvate carboxylase supports the pulmonary tropism of metastatic breast cancer. Breast Cancer Res. 2018;20(1):76.

14. Phannasil P, Thuwajit C, Warnnissorn M, Wallace JC, MacDonald MJ, Jitrapakdee S. Pyruvate Carboxylase Is Up-Regulated in Breast Cancer and Essential to Support Growth and Invasion of MDAMB-231 Cells. PLoS One. 2015;10(6):e0129848.

15. Christen S, Lorendeau D, Schmieder R, Broekaert D, Metzger K, Veys K, Elia I, Buescher JM, Orth MF, Davidson SM, et al. Breast Cancer-Derived Lung Metastases Show Increased Pyruvate CarboxylaseDependent Anaplerosis. Cell Rep. 2016;17(3):837-48.

16. Wilmanski T, Buhman K, Donkin SS, Burgess JR, Teegarden D. 1a,25-dihydroxyvitamin D inhibits de novo fatty acid synthesis and lipid accumulation in metastatic breast cancer cells through downregulation of pyruvate carboxylase. J Nutr Biochem. 2017;40:194-200.

17. Wilmanski T, Zhou X, Zheng W, Shinde A, Donkin SS, Wendt M, Burgess JR, Teegarden D. Inhibition of pyruvate carboxylase by 1a,25-dihydroxyvitamin D promotes oxidative stress in early breast cancer progression. Cancer Lett. 2017;411:171-81.

18. Long CP, Antoniewicz MR. High-resolution $13 \mathrm{C}$ metabolic flux analysis. Nat Protoc. 2019;14(10):2856-77.

19. Schafer ZT, Grassian AR, Song L, Jiang Z, Gerhart-Hines Z, Irie HY, Gao S, Puigserver P, Brugge JS. Antioxidant and oncogene rescue of metabolic defects caused by loss of matrix attachment. Nature. 2009;461(7260):109-13.

20. Endo H, Owada S, Inagaki Y, Shida Y, Tatemichi M. Metabolic reprogramming sustains cancer cell survival following extracellular matrix detachment. Redox Biol. 2020;36:101643.

21. Kiesel VA, Sheeley MP, Coleman MF, Cotul EK, Donkin SS, Hursting SD, Wendt MK, Teegarden D. Pyruvate carboxylase and cancer progression. Cancer Metab. 2021;9(1):20.

22. Sellers K, Fox MP, Bousamra M, Slone SP, Higashi RM, Miller DM, Wang Y, Yan J, Yuneva MO, Deshpande R, et al. Pyruvate carboxylase is critical for non-small-cell lung cancer proliferation. J Clin Invest. 2015;125(2):687-98.

23. Bergers G, Fendt SM. The metabolism of cancer cells during metastasis. Nat Rev Cancer 2021.

24. Cardaci S, Zheng L, MacKay G, van den Broek NJ, MacKenzie ED, Nixon C, Stevenson D, Tumanov S, Bulusu V, Kamphorst JJ, et al. Pyruvate carboxylation enables growth of SDH-deficient cells by supporting aspartate biosynthesis. Nat Cell Biol. 2015;17(10):1317-26. 
25. Lussey-Lepoutre C, Hollinshead KE, Ludwig C, Menara M, Morin A, Castro-Vega LJ, Parker SJ, Janin $\mathrm{M}$, Martinelli $\mathrm{C}$, Ottolenghi $\mathrm{C}$, et al. Loss of succinate dehydrogenase activity results in dependency on pyruvate carboxylation for cellular anabolism. Nat Commun. 2015;6:8784.

26. Lorendeau D, Rinaldi G, Boon R, Spincemaille P, Metzger K, Jäger C, Christen S, Dong X, Kuenen S, Voordeckers $\mathrm{K}$, et al. Dual loss of succinate dehydrogenase (SDH) and complex I activity is necessary to recapitulate the metabolic phenotype of SDH mutant tumors. Metab Eng. 2017;43(Pt B):187-97.

\section{Figures}



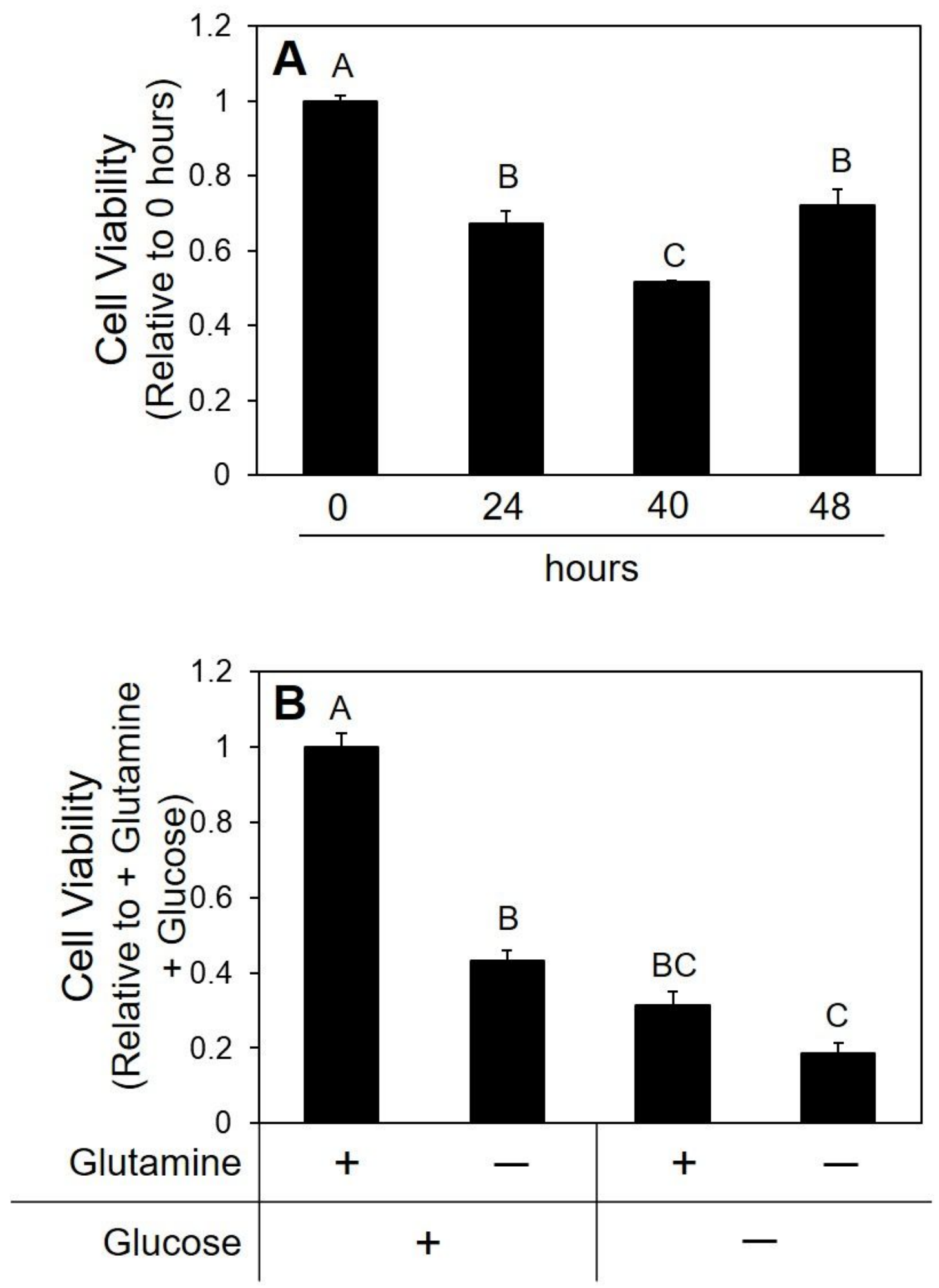

Figure 1

Effect of detachment on cell viability. (A) Viability of MCF10A-ras cells was assessed by MTT after 0,24 , 40 , and 48 hours in detached conditions, and (B) in glutamine and glucose depleted conditions. Results are expressed as \pm SEM. Different letters indicates significance $P<0.05$. 

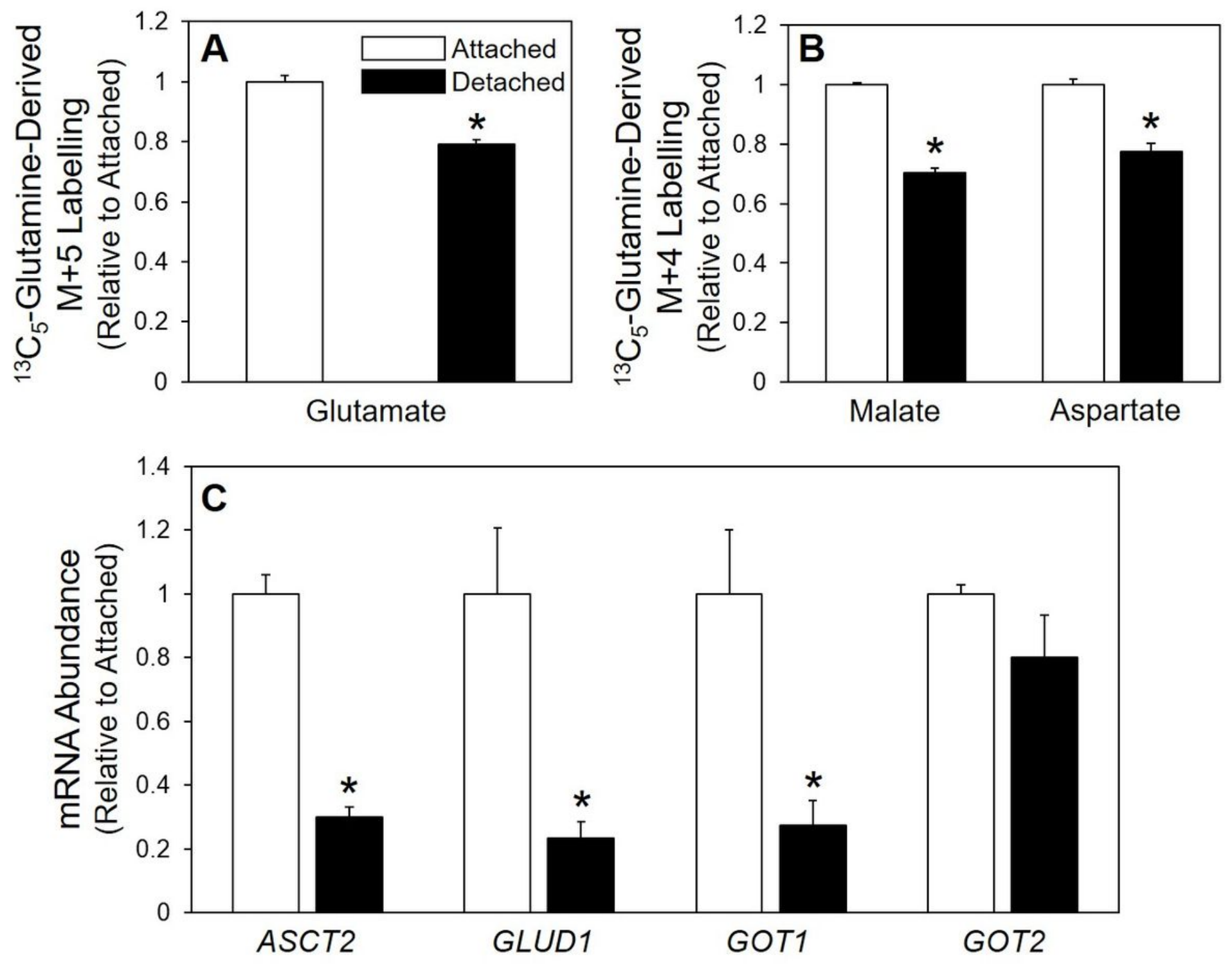

Figure 2

Glutamine metabolism in attached and detached conditions. (A) 13C5-Glutamine was used to determine $\mathrm{M}+5$ labeling of glutamate, and (B) M+4 malate and aspartate downstream metabolites in attached and detached MCF10A-ras cells. (C) mRNA abundance of enzymes in glutamine metabolism was assessed in attached and detached MCF10A-ras cells. Results are expressed as \pm SEM. Asterisk $\left(^{\star}\right)$ indicates $\mathrm{P}<0.05$ relative to attached cells. 

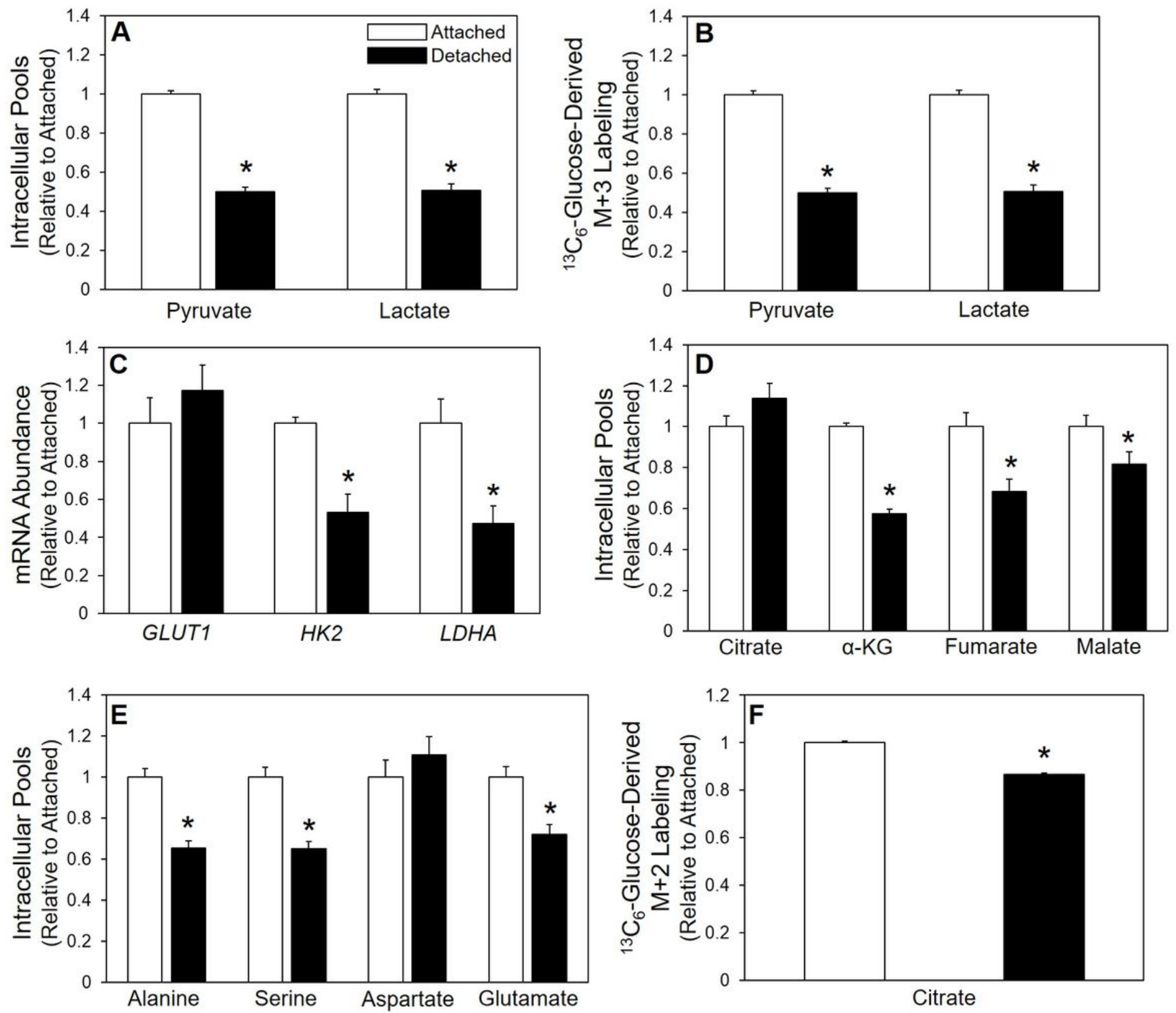

\section{Figure 3}

Glucose metabolism in attached and detached conditions. (A) Intracellular pools of pyruvate and lactate were measured and (B) 13C6-Glucose was used to determine $M+3$ labeling of pyruvate and lactate (C) mRNA abundance of enzymes in glucose metabolism was assessed (D) intracellular pools of citrate, aKG, fumarate, and malate were measured (E) intracellular pools of alanine, serine, aspartate, and glutamate were measured (F) 13C6-Glucose was used to determine $M+2$ labeling of citrate in attached and detached MCF10A-ras cells. Results are expressed as \pm SEM. Asterisk $\left(^{*}\right)$ indicates $P<0.05$ relative to attached cells. 

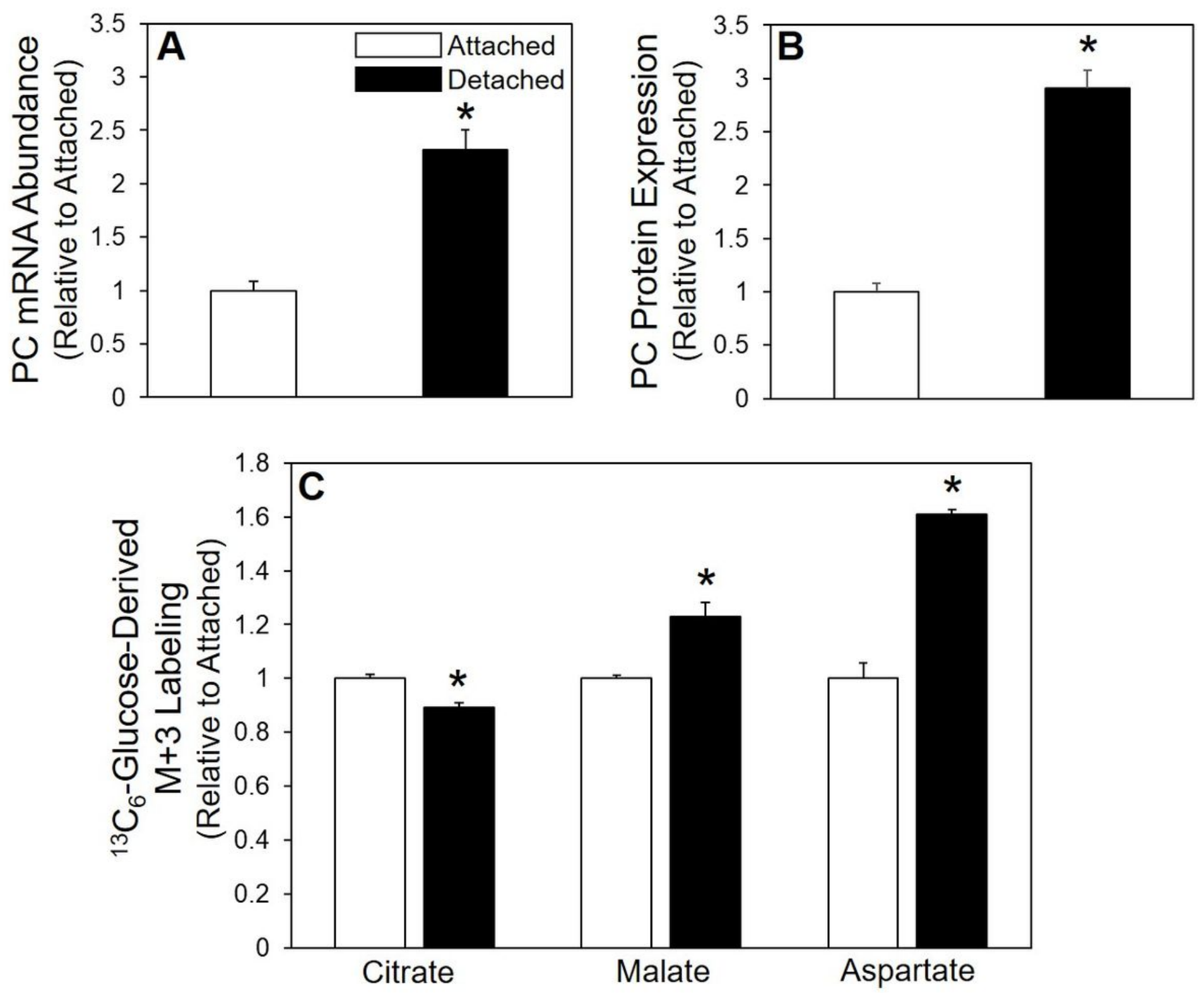

Figure 4

Effect of detachment on pyruvate carboxylase expression and activity. (A) mRNA abundance of PC was assessed (B) protein abundance of PC was assessed (C) PC activity determined from 13C6-Glucosederived $\mathrm{M}+3$ labeled citrate, malate, and aspartate in attached and detached MCF10A-ras cells. Results are expressed as \pm SEM. Asterisk $(*)$ indicates $\mathrm{P}<0.05$ relative to attached cells. 

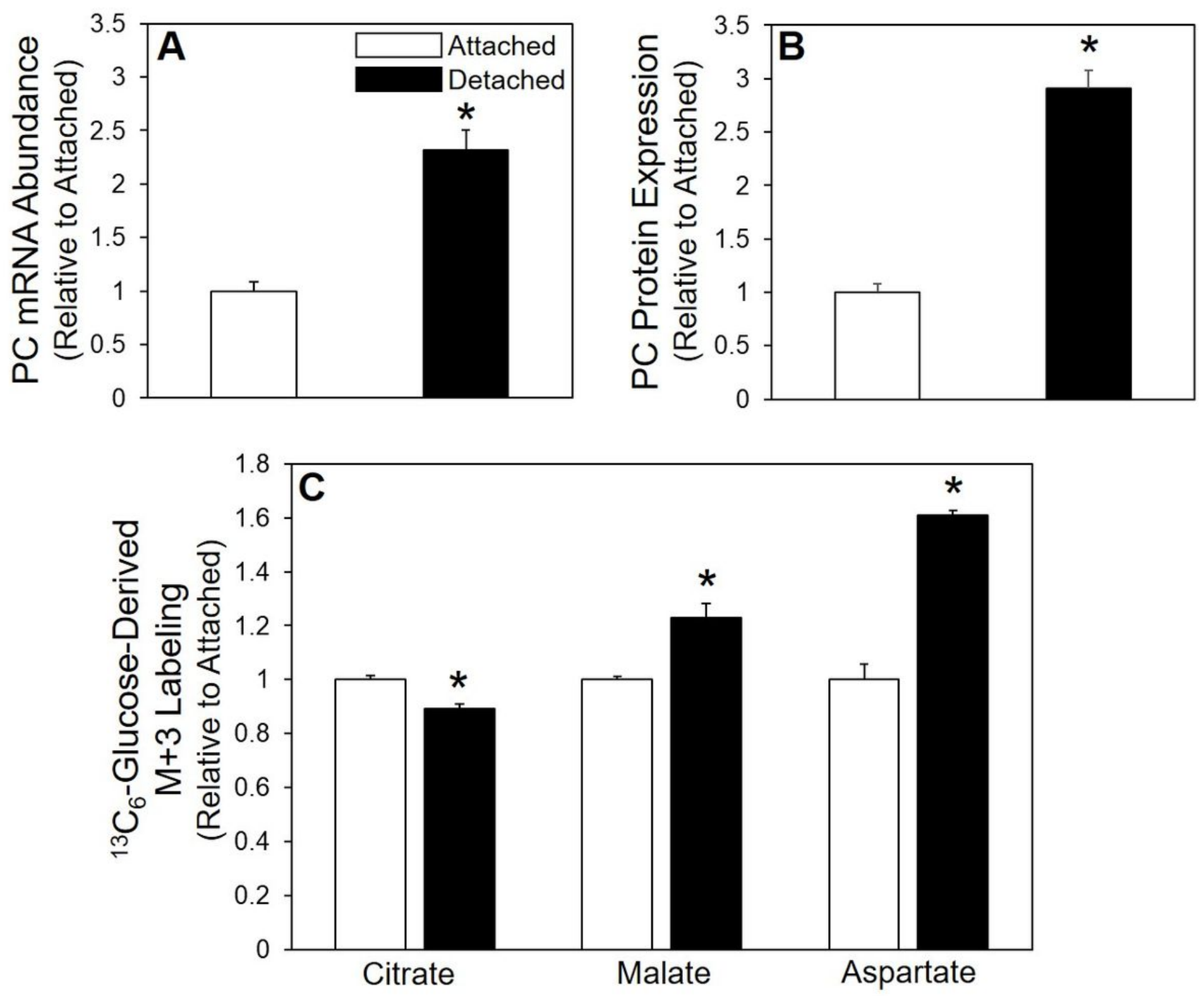

Figure 5

Detached cell viability with PC depletion. (A) mRNA abundance of PC in attached shPC Dox-inducible MCF10A-ras cells treated with and without $0.5 \mu \mathrm{g} / \mathrm{mL}$ Dox was assessed. (B) Viability with 2mM OAA and (C) $2.5 \mathrm{mM}$ Asp supplementation of detached Dox-inducible PC knockdown MCF10A-ras cells treated with and without Dox. Results are expressed as \pm SEM. Asterisk $\left(^{*}\right)$ or different letters indicates significance $\mathrm{P}<0.05$. 

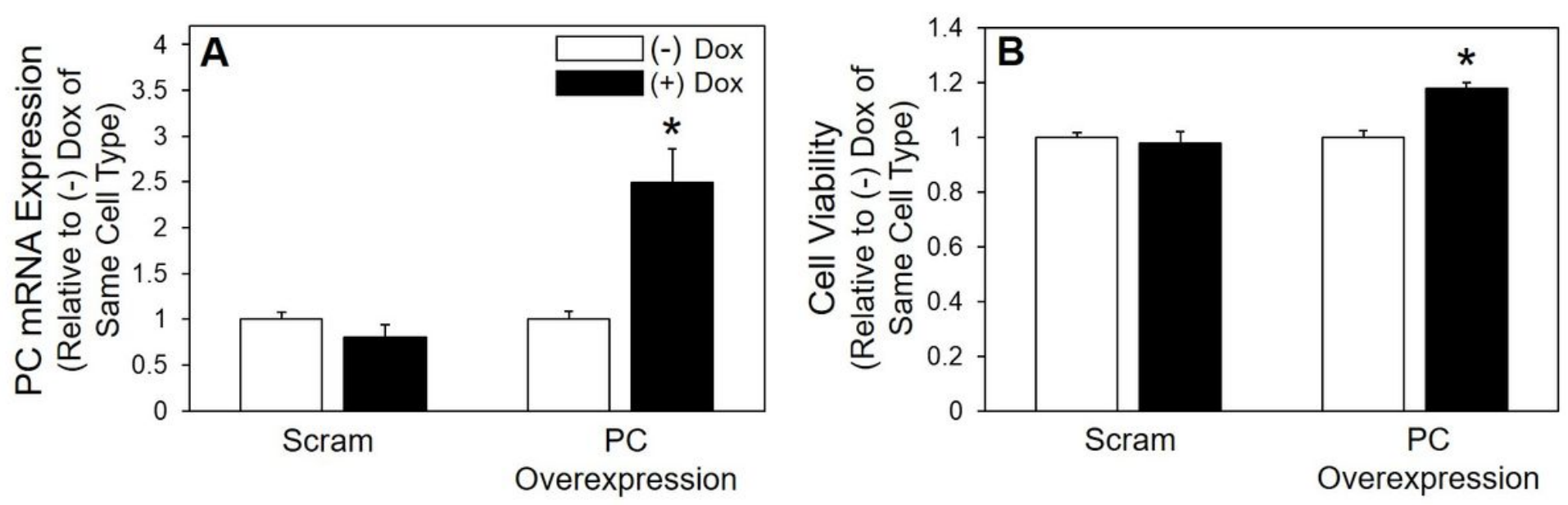

Figure 6

Effect of pyruvate carboxylase overexpression on detached cell viability. (A) mRNA abundance of PC of attached Dox-inducible PC overexpressing and Dox-inducible scram MCF10A-ras cells treated with 0.5 $\mu \mathrm{g} / \mathrm{mL}$ Dox was assessed. (B) Viability with and without Dox treatment of detached Dox-inducible PC overexpressing and Dox-inducible scram MCF10A-ras cells treated with and without Dox. Results are expressed as \pm SEM. Asterisk (*) indicates $\mathrm{P}<0.05$ relative to $(-)$ Dox of the same cell type.
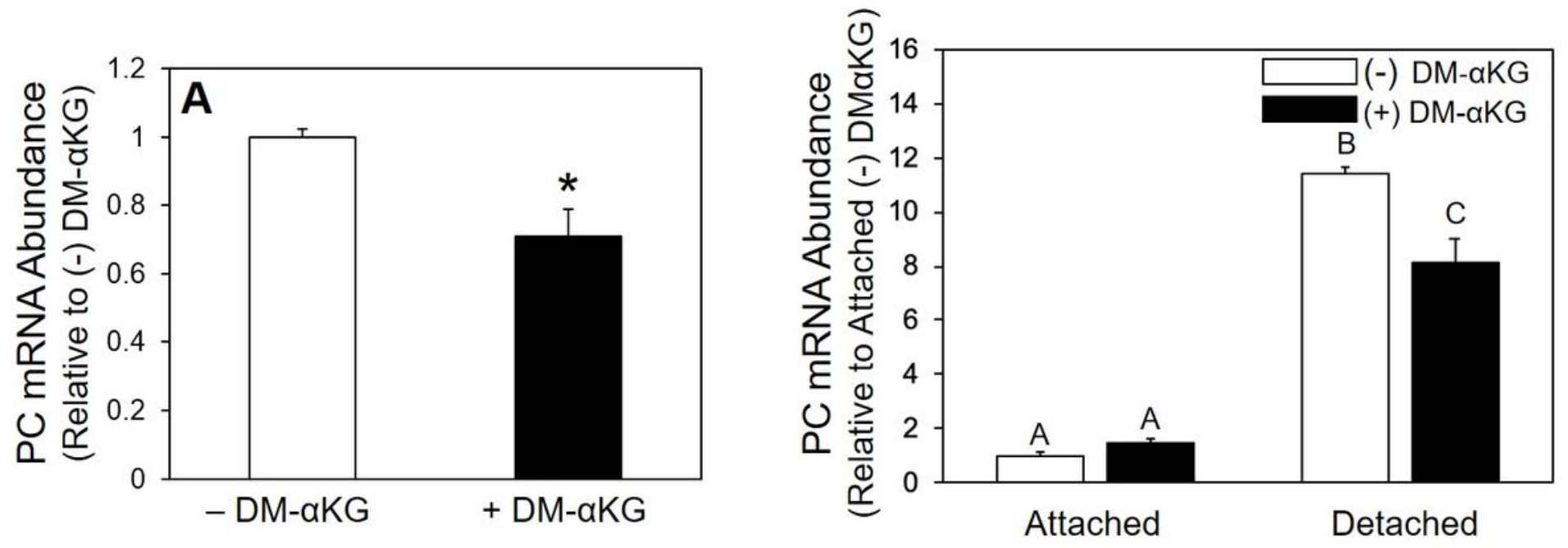

Figure 7

Dimethyl a-ketoglutarate's effect on PC expression. (A) mRNA abundance of PC of detached MCF10A-ras cells supplemented with $2 \mathrm{mM}$ DM-aKG was assessed. Results are expressed as \pm SEM. Asterisk $\left(^{\star}\right)$ indicates $\mathrm{P}<0.05$ relative to $(-)$ DM-aKG. 